\title{
Laser-induced dynamics of molecules with strong nuclear quadrupole coupling
}

Cite as: J. Chem. Phys. 151, 244118 (2019); https://doi.org/10.1063/1.5133837

Submitted: 27 October 2019 . Accepted: 03 December 2019 . Published Online: 27 December 2019

Andrey Yachmenev (D), Linda V. Thesing, and Jochen Küpper (D)
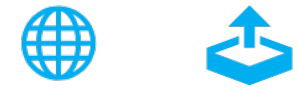

\section{ARTICLES YOU MAY BE INTERESTED IN}

Atomic-resolution imaging of carbonyl sulfide by laser-induced electron diffraction The Journal of Chemical Physics 150, 244301 (2019); https://doi.org/10.1063/1.5093959

Exact parameterization of fermionic wave functions via unitary coupled cluster theory The Journal of Chemical Physics 151, 244112 (2019); https://doi.org/10.1063/1.5133059

Vibronic structure and predissociation dynamics of 2-methoxythiophenol ( $\left.\mathrm{S}_{\uparrow}\right)$ : The effect of intramolecular hydrogen bonding on nonadiabatic dynamics

The Journal of Chemical Physics 151, 244305 (2019); https://doi.org/10.1063/1.5134519
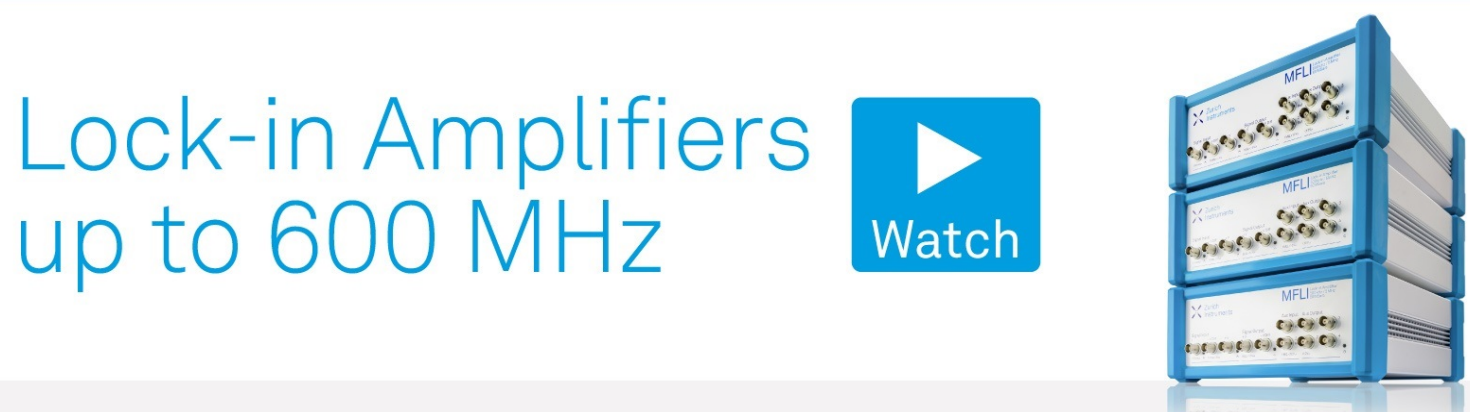


\title{
Laser-induced dynamics of molecules with strong nuclear quadrupole coupling
}

\author{
Cite as: J. Chem. Phys. 151, 244118 (2019); doi: 10.1063/1.5133837 \\ Submitted: 27 October 2019 - Accepted: 3 December 2019 • \\ Published Online: 27 December 2019

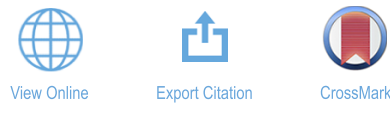

\author{
Andrey Yachmenev, ${ }^{1,2, a)}$ (D) Linda V. Thesing, ${ }^{1,2,3}$ and Jochen Küpper ${ }^{1,2,3}$ (D)
}

\begin{abstract}
AFFILIATIONS
${ }^{1}$ Center for Free-Electron Laser Science, Deutsches Elektronen-Synchrotron DESY, Notkestraße 85, 22607 Hamburg, Germany

${ }^{2}$ Center for Ultrafast Imaging, Universität Hamburg, Luruper Chaussee 149, 22761 Hamburg, Germany

${ }^{3}$ Department of Physics, Universität Hamburg, Luruper Chaussee 149, 22761 Hamburg, Germany
\end{abstract}

a)Electronic mail: andrey.yachmenev@cfel.de. URL: https://www.controlled-molecule-imaging.org.

\begin{abstract}
We present a general variational approach for computing the laser-induced rovibrational dynamics of molecules, taking into account the hyperfine effects of the nuclear quadrupole coupling. The method combines the general variational approach TROVE (Theoretical RoVibrational Energies), which provides accurate rovibrational hyperfine energies and wavefunctions for arbitrary molecules, with the variational method RichMol, designed for generalized simulations of the rovibrational dynamics in the presence of external electric fields. We investigate the effect of the nuclear quadrupole coupling on the short-pulse laser alignment of a prototypical molecule CFClBrI, which contains nuclei with large quadrupole constants. The influence of the nuclear quadrupole interactions on the postpulse molecular dynamics is negligible at early times, for the first several revivals; however, at longer time scales, the effect is entirely detrimental and strongly depends on the laser intensity. This effect can be explained by dephasing in the laser-excited rotational wavepacket due to irregular spacings between the hyperfine-split nuclear spin states across different rotational hyperfine bands.
\end{abstract}

Published under license by AIP Publishing. https://doi.org/10.1063/1.5133837

Laser-controlled rovibrational molecular dynamics is a subject of active research in physics and chemistry. ${ }^{1-4}$ In particular, the control of molecular spatial alignment and orientation is highly leveraged in many ultrafast imaging experiments ${ }^{3,8-14}$ and stereochemistry studies ${ }^{15-17}$ to increase the experimental resolution. The mechanism of the alignment and orientation is tied to the driven rotational dynamics of molecules in the ground vibrational state and described by two-photon ${ }^{18,19}$ or threephoton $^{20,21}$ Raman excitation processes. The adiabatic alignment is induced by slowly increasing the laser electric field, which creates directional potential trapping molecules in pendular states. Adiabatic alignment combined with a DC electric field produces the mixed-field orientation of molecules. ${ }^{6,22,23}$ In the impulsive alignment regime using short laser pulses, the created rotational wavepacket evolves with repeated alignment/orientation and antialignment revivals. In all of these schemes, the temporal evolution of the rotational wavepacket and the resulting revival structure is characteristic of the rotational-energy-level structure of the molecule. ${ }^{23-25}$ Typically, this temporal wavepacket evolution is accurately predicted using the rigid-rotor or semirigid rotor Hamiltonian models. ${ }^{5,26-28}$

Many heavy atoms, such as bromine, iodine, or platinum, have large nuclear quadrupoles. In molecules, these create large hyperfine splittings, comparable to or even larger than the rotationalenergy spacings. These strong hyperfine interactions arise from the coupling between the quadrupole moments of nuclei and the electric field gradients produced by the distribution of nuclei and electrons through the molecule. In such heavy-atom containing molecules, the presence of the manifold of hyperfine-split nuclear-spin states makes the temporal evolution of the laserexcited rotational wavepacket, and thus the alignment revival structure, more complicated. ${ }^{29}$ Therefore, a detailed understanding of the effect of these nuclear-quadrupole interactions is an important ingredient for the control over the alignment of heavyatom-containing molecules, with corresponding implications for molecular-frame imaging experiments. Heavy atoms are commonly utilized as strong scattering and absorption centers in x-ray imaging experiments. ${ }^{9,31}$ They are also exploited as good leaving 
groups in Coulomb-explosion velocity-map imaging of molecular dynamics. ${ }^{32,33}$

Here, we present a general variational approach for computing the field-driven rovibrational dynamics of molecules including nuclear-quadrupole interactions. The present approach extends our previously reported variational method for computing the nuclearquadrupole hyperfine spectra of small molecules. ${ }^{34}$ We developed a generalized methodology for computing the matrix representations of various electric-multipole-moment tensor operators in the basis of hyperfine wavefunctions. These tensor operators are used as building blocks of the molecule-field interaction potential in simulations of the field-driven rovibrational dynamics, as implemented in the computational approach RichMol. ${ }^{35}$ To our knowledge, this is the first attempt to generalize simulations of this kind. We demonstrate the effect by calculating the one-dimensional alignment dynamics of the asymmetric $\mathrm{CF}^{35} \mathrm{Cl}^{79} \mathrm{Br}^{127} \mathrm{I}$ molecule by a short laser pulse.

In brief, in RichMol, the time-dependent wavepacket $\Psi(t)$ is built from a superposition of the field-free rovibrational wavefunctions $\left|J, m_{J}, l\right\rangle$,

$$
\Psi(t)=\sum_{J, m_{J}, l} c_{J, m_{J}, l}(t)\left|J, m_{J}, l\right\rangle,
$$

where $J$ and $m_{J}$ denote the quantum numbers of the total rotational angular momentum operator $\mathbf{J}$ and its projection onto the laboratory-fixed $Z$ axis, respectively. $l$ represents a set of additional rotational and vibrational quantum numbers. The time-dependent coefficients $c_{J, m_{J}, l}(t)$ are determined by a numerical solution of the time-dependent Schrödinger equation using the time-evolution operator method. The total Hamiltonian consists of the sum of the molecular rovibrational Hamiltonian $H_{\mathrm{rv}}$, with the eigenfunctions $\left|J, m_{J}, l\right\rangle$, and the molecule-field interaction potential $V(t)$, expanded in terms of molecular electric multipole moment operators,

$$
H(t)=H_{\mathrm{rv}}-\mu_{A} E_{A}(t)-\frac{1}{2} \alpha_{A B} E_{A}(t) E_{B}(t)+\cdots .
$$

$A$ and $B$ are Cartesian indices denoting the $X, Y$, and $Z$ axes in the laboratory frame, and the summation over all Cartesian indices is implicitly assumed. $E_{A}(t)$ is the $A$ Cartesian component of the electric field vector, and $\mu_{A}$ and $\alpha_{A B}$ are the electronic contributions to the molecular-frame electric-dipole-moment vector and polarizability tensor, respectively. The interaction terms of the higher expansion order, such as the first and second hyperpolarizability tensors, can also, in principle, be included in the sum in (2).

The time-evolution operator for the time step $\Delta t=t-t^{\prime}$ is computed using the split-operator method as

$$
\mathcal{U}\left(t, t^{\prime}\right)=e^{-i \frac{\Delta t}{2 \hbar} H_{\mathrm{rv}}}\left(e^{i \frac{\Delta t}{\hbar} \mu_{A} E_{A}\left(\frac{t+t^{\prime}}{2}\right)} \cdot e^{i \frac{\Delta t}{2 \hbar} \alpha_{A B} E_{A}\left(\frac{t+t^{\prime}}{2}\right) E_{B}\left(\frac{t+t^{\prime}}{2}\right)} \cdot \ldots\right) e^{-i \frac{\Delta t}{2 \hbar} H_{\mathrm{rv}}} .
$$

$H_{\mathrm{rv}}$ is diagonal in the basis of its eigenfunctions $\left|J, m_{J}, l\right\rangle$, and the diagonal elements are the molecular rovibrational energies. The exponentials of the Cartesian tensor operators $\mu_{A}, \alpha_{A B}, \ldots$ are evaluated using an iterative approximation based on the Krylov-subspace methods. The computational performance of the iterative methods depends crucially on how efficient the matrix-vector products can be computed between the operator exponential and the subspace vectors. In the following, we will use $T_{A}^{(\Omega)}$ to denote any index-symmetric Cartesian tensor operator of $\operatorname{rank} \Omega$, with $A$ being multi-index labeling Cartesian components in the upper simplex of the tensor in the laboratory frame. For example, for the dipole moment, $\Omega=1$ and $A=X, Y$, or $Z$, and for the polarizability tensor, $\Omega=2$ and $A=X X, X Y, X Z, Y Y, Y Z$, or $Z Z$.

The computations of the matrix-vector products can be significantly sped up by expressing the matrix representation of Cartesian tensor operators $T_{A}^{(\Omega)}$ in a contracted tensor form,

$$
\left\langle J^{\prime}, m_{J}^{\prime}, l^{\prime}\left|T_{A}^{(\Omega)}\right| J, m_{J}, l\right\rangle=\sum_{\omega=0}^{\Omega} \mathcal{M}_{A, \omega}^{\left(J^{\prime}, m_{J}^{\prime}, J, m_{J}\right)} \mathcal{K}_{\omega}^{\left(J^{\prime}, l^{\prime}, J, l\right)} .
$$

The sum runs over all irreducible representations $\omega$ of the tensor, and the matrices $\mathcal{M}_{A, \omega}^{\left(J^{\prime}, m_{J}^{\prime}, J, m_{J}\right)}$ and $\mathcal{K}_{\omega}^{\left(J^{\prime}, l^{\prime}, J, l\right)}$ decouple the laboratoryframe projections $A$ and quantum numbers $m_{J}$ and $m_{J}^{\prime}$ from the molecular-frame rovibrational quantum numbers $l$ and $l^{\prime}$.

The explicit expressions for the $\mathcal{M}_{A, \omega}$ and $\mathcal{K}_{\omega}$ matrices depend on the form of the field-free wavefunctions $\left|J, m_{J}, l\right\rangle$. We use the general-molecule variational approach TROVE (Theoretical RoVibrational Energies) ${ }^{36-39}$ to compute the field-free energies and wavefunctions $\left|J, m_{J}, l\right\rangle$, which are represented by linear combinations of products of vibrational wavefunctions $|v\rangle$ and symmetrictop rotational functions $\left|J, m_{J}, k\right\rangle$,

$$
\left|J, m_{J}, l\right\rangle=\sum_{v, k} c_{v, k}^{(J, l)}|v\rangle\left|J, m_{J}, k\right\rangle
$$

$v$ denotes the composite vibrational quantum number, and $k$ denotes the quantum number of the molecular-frame $z$-projection of the rotational angular momentum operator. Using the wavefunctions from (5), the expressions for $\mathcal{M}_{A, \omega}$ and $\mathcal{K}_{\omega}$ can be derived as

$$
\begin{aligned}
\mathcal{M}_{A, \omega}^{\left(J^{\prime}, m_{J}^{\prime}, J, m_{J}\right)}= & (-1)^{m_{J}^{\prime}} \sqrt{\left(2 J^{\prime}+1\right)(2 J+1)} \\
& \times \sum_{\sigma=-\omega}^{\omega}\left[U^{(\Omega)}\right]_{A, \omega, \sigma}^{-1}\left(\begin{array}{ccc}
J & \omega & J^{\prime} \\
m_{J} & \sigma & -m_{J}^{\prime}
\end{array}\right)
\end{aligned}
$$

and

$$
\begin{aligned}
\mathcal{K}_{\omega}^{\left(J^{\prime}, l^{\prime}, J, l\right)}= & \sum_{k^{\prime}, v^{\prime}} \sum_{k, v}\left[c_{v^{\prime}, k^{\prime}}^{\left(J^{\prime}, l^{\prime}\right)}\right]^{*} c_{v, k}^{(J, l)}(-1)^{k^{\prime}} \\
& \times \sum_{\sigma=-\omega}^{\omega} \sum_{a}\left(\begin{array}{ccc}
J & \omega & J^{\prime} \\
k & \sigma & -k^{\prime}
\end{array}\right) U_{\omega, \sigma, a}^{(\Omega)}\left\langle v^{\prime}\left|T_{a}^{(\Omega)}\right| v\right\rangle .
\end{aligned}
$$

$T_{a}^{(\Omega)}$ denotes the Cartesian tensor operator in the molecular frame, with $a$ being a Cartesian multi-index (similar to $A$ ), and the matrix $U^{(\Omega)}$ defines the transformation of the tensor from Cartesian to spherical-tensor form.

This approach is general and can be interfaced with any rovibrational computer code that provides the tensor-matrix elements in the form of (4). The approach also permits the use of more complex field-free wavefunctions than those defined in (5). As multipole-moment operators commute with the nuclear-spin angular momenta, the operators' matrix elements in the basis of hyperfine states can also be cast in the form of (4). In the following, we derive the explicit expressions for the $\mathcal{M}_{A, \omega}$ and $\mathcal{K}_{\omega}$ matrix elements in the basis of the nuclear-spin hyperfine-structure wavefunctions.

The general variational implementation of the nuclearspin hyperfine effects at the level of the nuclear-quadrupole 
interaction was recently implemented ${ }^{34}$ and used for the generation of a quadrupole-resolved line list of the ammonia molecule. ${ }^{40}$ The nuclear-quadrupole interaction in a molecule containing $n=1 \ldots N$ quadrupolar nuclei is described by the coupling of the electric field gradient (EFG) tensor at each $n$th nucleus $\mathbf{V}(n)$ with its quadrupole moment tensor $\mathbf{Q}(n)$. The total spin-rovibrational Hamiltonian takes the form

$$
H_{\mathrm{srv}}=H_{\mathrm{rv}}+\sum_{n} \mathbf{V}(n) \cdot \mathbf{Q}(n) .
$$

The overall rotational, $\mathbf{J}$, the nuclear spin, $\mathbf{I}_{n}$, and total, $\mathbf{F}$, angular momentum operators for $n=1 \ldots N$ quadrupolar nuclei are coupled as $\mathbf{I}_{1,2}=\mathbf{I}_{1}+\mathbf{I}_{2}, \mathbf{I}_{1,3}=\mathbf{I}_{1,2}+\mathbf{I}_{3}, \ldots, \mathbf{I}_{1, N-1}=\mathbf{I}_{1, N-2}+\mathbf{I}_{N-1}$, $\mathbf{I} \equiv \mathbf{I}_{1, N}=\mathbf{I}_{1, N-1}+\mathbf{I}_{N}$, and $\mathbf{F}=\mathbf{J}+\mathbf{I}$. The nuclear-spin functions $\left|I, m_{I}, \mathcal{I}\right\rangle$ depend on the quantum numbers $I$ and $m_{I}$ of the collective nuclear spin angular momentum operator $I$ and its projection onto the laboratory $Z$ axis, respectively. The set of auxiliary spin quantum numbers $\mathcal{I}=\left\{I_{1}, I_{1,2}, \ldots, I_{1, N-1}\right\}$ of the intermediate spin angular momentum operators provide a unique assignment of each nuclearspin state. The total spin-rovibrational wavefunctions $\left|F, m_{F}, u\right\rangle$ are built as symmetry-adapted linear combinations of the products of the rovibrational wavefunctions $\left|J, m_{J}, l\right\rangle$ in (5) and the nuclear-spin functions $\left|I, m_{I}, \mathcal{I}\right\rangle$,

$$
\begin{aligned}
\left|F, m_{F}, u\right\rangle= & \sum_{I, \mathcal{I}, J, l} c_{I, \mathcal{I}, J, l}^{(F, u)}\left[\sum_{m_{J}, m_{I}}(-1)^{F+m_{F}} \sqrt{2 F+1}\right. \\
& \left.\times\left(\begin{array}{ccc}
F & I & J \\
-m_{F} & m_{I} & m_{J}
\end{array}\right)\left|I, m_{I}, \mathcal{I}\right\rangle\left|J, m_{J}, l\right\rangle\right] .
\end{aligned}
$$

$c_{I, \mathcal{I}, J, l}^{(F, u)}$ are the eigenvector coefficients of the spin-rovibrational Hamiltonian (8), $F$ and $m_{F}$ are the quantum numbers of $\mathbf{F}$ and its projection onto the laboratory-fixed $Z$-axis, and $u$ denotes the hyperfine-state running index.

The explicit expressions for the matrix elements of the spinrovibrational Hamiltonian (8) for an arbitrary number of quadrupolar nuclei and details of the variational solution can be found in Ref. 34. Here, we derived explicit expressions for the matrix elements of the general electric multipole Cartesian tensor operator $\left\langle F^{\prime}, m_{F}^{\prime}, u^{\prime}\left|T_{A}^{(\Omega)}\right| F, m_{F}, u\right\rangle$, which, in the contracted-tensor form of (4), are

$$
\begin{aligned}
\mathcal{M}_{A, \omega}^{\left(F^{\prime}, m_{F}^{\prime}, F, m_{F}\right)}= & (-1)^{m_{F}^{\prime}} \sqrt{\left(2 F^{\prime}+1\right)(2 F+1)} \\
& \times \sum_{\sigma=-\omega}^{\omega}\left[U^{(\Omega)}\right]_{A, \omega, \sigma}^{-1}\left(\begin{array}{ccc}
F^{\prime} & \omega & F \\
-m_{F}^{\prime} & \sigma & m_{F}
\end{array}\right)
\end{aligned}
$$

and

$$
\begin{aligned}
\mathcal{K}_{\omega}^{\left(F^{\prime}, u^{\prime}, F, u\right)}= & \sum_{I^{\prime}, \mathcal{I}^{\prime}, J^{\prime}, l^{\prime}} \sum_{I, \mathcal{I}, J, l^{\prime}}\left[c_{I^{\prime}, \mathcal{I}^{\prime}, J^{\prime}, l^{\prime}}\right]^{F^{\prime}, c^{\prime}} c_{I, \mathcal{I}, J, l}^{(F, u)}(-1)^{I} \\
& \times \sqrt{\left(2 J^{\prime}+1\right)(2 J+1)}\left\{\begin{array}{lll}
J^{\prime} & F^{\prime} & I \\
F & J & \omega
\end{array}\right\} \\
& \times \mathcal{K}_{\omega}^{\left(J^{\prime}, l^{\prime}, J, l\right)} \delta_{I^{\prime}, I} \delta_{\mathcal{I}^{\prime}, \mathcal{I}} .
\end{aligned}
$$

$\mathcal{K}_{\omega}^{\left(J^{\prime}, l^{\prime}, J, l\right)}$, defined in (7), contains the rovibrational matrix elements of $T_{A}^{(\Omega)}$ in the basis $\left|J, m_{J}, l\right\rangle$. Using the expressions (10) and (11) in (4), RichMol could directly be employed to simulate the coupled nuclear-spin-rovibrational molecular dynamics in external fields. In (3), the diagonal representation of $H_{\mathrm{rv}}$ in the rovibrational energies was replaced by the diagonal representation of $H_{\text {srv }}$ in the hyperfine energies (8).

This new approach was used to investigate the effect of nuclearquadrupole coupling (QC) on the impulsive alignment of bromochlorofluoroiodomethane $\mathrm{CF}^{35} \mathrm{Cl}^{79} \mathrm{Br}^{127} \mathrm{I}$. This molecule has a quasirigid structure and contains three different heavy nuclei with large quadrupole coupling constants and with correspondingly nontrivial laser-induced rotational and nuclear spin spectra and dynamics. A short nonresonant laser pulse is linearly polarized along the laboratory $Z$ axis. Its intensity is given by the Gaussian function $I(t)=I_{0} \exp \left(-4 \ln 2 t^{2} / \sigma^{2}\right)$ with $\sigma=1$ ps and $I_{0}=6$ $\times 10^{11} \mathrm{~W} / \mathrm{cm}^{2}$ or $1 \times 10^{12} \mathrm{~W} / \mathrm{cm}^{2}$. The excitation by a nonresonant laser field is described by the electric polarizability term in the interaction potential (2). The explicit expressions for the elements of the matrix $U^{(2)}$ for the polarizability are listed in Table I of Ref. 35. The degree of molecular alignment is characterized by the expectation value $\left\langle\cos ^{2} \theta\right\rangle=\left\langle\Psi(t)\left|\cos ^{2} \theta\right| \Psi(t)\right\rangle$, with the angle $\theta$ between the molecular-frame $z$ and the laboratory-frame $Z$ axes. The matrix elements of the $\cos ^{2} \theta$ operator can be easily computed using the general expressions (10), (11), and (7) by noting the relationship $\cos ^{2} \theta=\left(2 d_{00}^{2}+1\right) / 3$, where $d$ is the Wigner $d$ matrix. The vibrational matrix elements of $d_{00}^{2}$ in (7) are $\left\langle v^{\prime}\left|d_{00}^{2}\right| v\right\rangle=\delta_{v^{\prime} v}$ and $U_{\omega, \sigma}^{(0)}=\delta_{\omega, 2} \delta_{\sigma, 0}$; here, the index $a$ in (7) is redundant.

For simplicity, we neglected the vibrational motion of the molecule and approximated the full rovibrational wavefunctions in (5) by rigid-rotor solutions. The rigid-rotor approximation in simulations of the laser-induced alignment of quasirigid and even nonrigid molecules in ultracold molecular beams has been validated in numerous studies. ${ }^{23,28,41-44}$ The zero-point vibrational corrections to various electromagnetic tensors are known to be quite small, on the order of $1 \%-3 \%$, even for nonrigid molecules such as $\mathrm{H}_{2} \mathrm{O}_{2},{ }^{45}$ and have been neglected in this study. The equilibrium geometry and polarizability of CFClBrI were calculated using density functional theory (DFT) with the B3LYP functional and the def2-TZVPP basis set $^{46,47}$ in conjunction with the relativistic effective core potential def2-ECP on the iodine atom. ${ }^{48}$ The accuracy of hybrid functionals for the prediction of molecular polarizabilities was assessed on a dataset of 132 molecules, yielding a root-mean-square error of $3 \%-5 \%$ relative to coupled-cluster singles and doubles with a perturbative correction to triples $[\mathrm{CCSD}(\mathrm{T})]] .{ }^{49}$ Calculations of the electric field gradient tensors, needed for the nuclear-quadrupole coupling Hamiltonian in (8), were carried out at the DFT/B3LYP level of theory using the all-electron scalar relativistic Douglas-Kroll-Hess Hamiltonian $^{50}$ with the DKH-def2-TZVP basis set. ${ }^{51,52}$ Systematic studies of the accuracy of DFT functionals for predictions of the electric field gradient tensors of transition metal complexes provided a mean error estimate of about 0.071 a.u. for the B3LYP functional. ${ }^{53}$ All electronic structure calculations employed the quantum chemistry package ORCA. ${ }^{54,55}$ The quadrupole moments for the ${ }^{35} \mathrm{Cl}$, ${ }^{79} \mathrm{Br}$, and ${ }^{127} \mathrm{I}$ nuclei are $Q=-81.65 \mathrm{mb}, 313 \mathrm{mb}$, and $-696 \mathrm{mb}$, respectively.

The calculations of the molecule-field dynamics in the basis of the nuclear-spin hyperfine states were performed in a three-step 


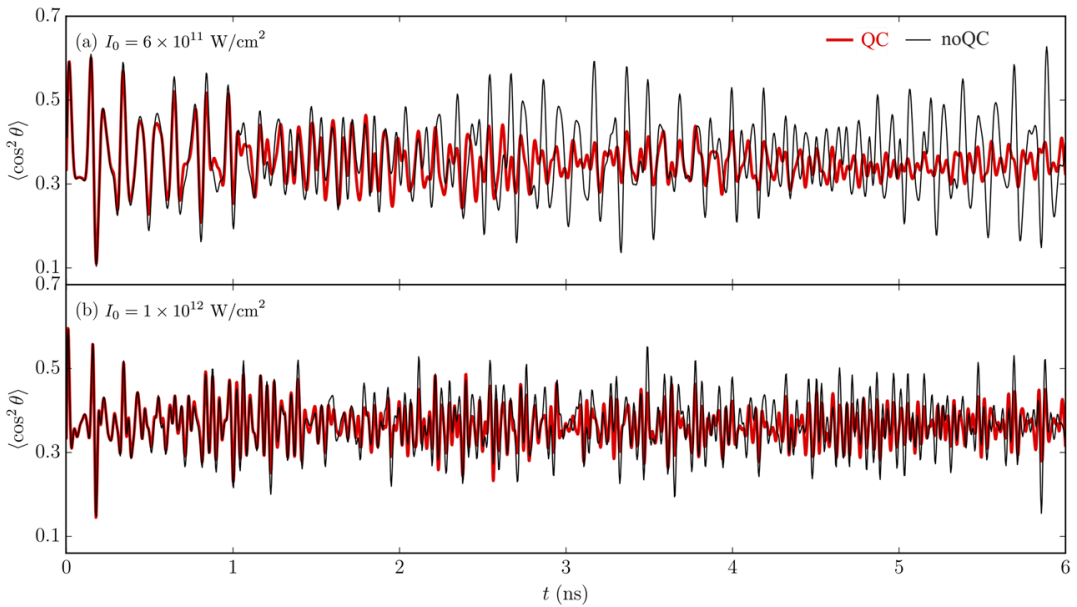

FIG. 1. Laser-alignment dynamics of $\mathrm{CFClBrl}$ with and without nuclearquadrupole coupling (QC and noQC) following excitation by a Gaussian laser pulse with $\tau_{\mathrm{FWHM}}=1 \mathrm{ps}$ and a maximum laser intensity of (a) $I_{0}=6 \times 10^{11} \mathrm{~W} / \mathrm{cm}^{2}$ and (b) $I_{0}=1 \times 10^{12} \mathrm{~W} / \mathrm{cm}^{2}$ procedure: First, we solved the field-free problem using the rigidrotor Hamiltonian and obtained the rotational energies and the rotational matrix elements of the EFG and the polarizability tensors, as well as of the $\cos ^{2} \theta$ operator, (6) and (7). Then, we used the rotational energies and the matrix elements of the EFG tensors together with the nuclear-quadrupole constants to build and diagonalize the nuclear-spin quadrupole-coupling Hamiltonian, ${ }^{34}$ (8). We obtained the spin-rotational eigenfunctions in (9) and transformed the rotational matrix elements of the polarizability tensor and $\cos ^{2} \theta$ operator into the spin-rotational eigenbasis using (10) and (11). Finally, we used the spin-rotational energies and the matrix elements of the polarizability to build the moleculefield interaction Hamiltonian and solve the time-dependent problem. The wavepacket $\Psi(t)$ in (1) was built from a linear combination of spin-rotational wavefunctions with time-dependent coefficients. The expectation value $\left\langle\cos ^{2} \theta\right\rangle$ is calculated from the spin-rotational matrix elements of $\cos ^{2} \theta$ computed at the previous step.

Here, we used a rotational basis that included all spin-rotational states of CFClBrI with $F \leq 41 / 2$, corresponding to $J \leq 26$. We assumed that $\mathrm{CFClBrI}$ is initially in the ground rotational state $J=0$, which has 96 different nuclear-spin state components, including the $m_{F}$ degeneracy. Therefore, we performed a series of simulations starting from the different nuclear-spin components and averaged the results assuming equal normalized populations of different spin components. For comparison, we also performed calculations neglecting the hyperfine effects, i.e., using rigid-rotor wavefunctions as the field-free basis. It should be noted that samples of heavy molecules populating a few of the lowest rotational states, with $T_{\text {rot }} \approx 0.4 \mathrm{~K}$, can be produced from cold molecular beams using the electrostatic deflector.

The temporal evolution of the alignment calculated for two different laser intensities is shown in Fig. 1. For both laser intensities, the alignment without nuclear-quadrupole interaction shows complex revival patterns originating from the dephasing and rephasing of the rotational wavepacket. Since $\mathrm{CFClBrI}$ is an asymmetrictop molecule (asymmetry parameter $\kappa=-0.75$ ), the revival patterns lack the typical periodicity of the $J$-type or $C$-type revivals observed for near-symmetric-top molecules. ${ }^{42,60,61}$ Notably, the broader wavepacket, produced by the higher-intensity pulse, shows higher-frequency oscillations and generally reduced peak alignment. This is ascribed to mismatches of the phases of the populated rotational states after the laser pulse ${ }^{62}$ and successive dephasing due to the molecule's asymmetry, ${ }^{42}$ preventing the simultaneous rephasing of more than a few rotational states with significant populations.

The nuclear-quadrupole interaction increases the complexity of the revival dynamics and depletion of the peak alignment compared to the rigid-rotor results. Nevertheless, during the first 300 ps after the laser pulse, the degree of alignment is almost identical to the rigid-rotor result. We also observed a dependence of the nuclearquadrupole effect on the laser-field intensity. For the lower intensity, Fig. 1(a), the impact is seen about 200 ps earlier than for the higher intensity, Fig. 1(b).

The nonperiodic recurrences in the alignment dynamics of the asymmetric-top molecules originate from large asymmetry splittings of the rotational energy levels. The influence of the nuclearquadrupole interaction can be understood in a similar manner, as the dephasing effect resulting from the incommensurate hyperfine splittings for different rotational states. The dephasing is stronger for the wavepacket dominated by the low-energy rotational states, where the hyperfine splittings are mostly irregular. For large $J$, the hyperfine splittings become increasingly uniform ${ }^{63}$ and the contribution to the dephasing is minimized. This explains why the effect of the nuclear-quadrupole interaction is less striking for the higherintensity laser field, which populates higher-energy rotational states. For both intensities, however, the small- $J$ states have relatively large populations and the nuclear-quadrupole interaction has a strong influence on the field-free alignment.

In conclusion, we have presented the first general implementation of nuclear-quadrupole hyperfine effects in the rovibrational dynamics of molecules driven by external electric fields. Our approach combines TROVE, which provides hyperfine energies and wavefunctions for the arbitrary molecule, with RichMol, designed for generalized simulations of external-field effects. In principle, the presented approach can be applied to simulate the fully coupled spin-rovibrational dynamics of any molecule with no inherent limitations on the number of quadrupolar nuclei. The external field 
effects are not limited by the polarizability interaction: other multipole moment operators, including the permanent dipole moment or the first and second hyperpolarizabilities, can be considered without additional implementation efforts.

We studied the influence of the nuclear-quadrupole coupling on the laser impulsive alignment of a prototypical heavy-atom molecule CFClBrI. While the effect is small for the first few revivals, it turns out to be entirely detrimental for the revivals at later times. The laser field plays an important role, with lower intensities prompting a larger effect of the nuclear-quadrupole coupling. This can be explained by dephasing of the rotational wavepacket due to the incommensurate structure of the hyperfine-split levels for different rotational states. This effect is stronger for small- $J$ rotational states and practically disappears for states with high angular momenta. Given that small- $J$ states in the wavepacket will be the source of the largest dephasing effects, we expect that stronger laser intensities and higher initial rotational temperatures should further diminish the effect of the nuclear-quadrupole coupling. More rigorous studies of these effects for different alignment scenarios and molecular systems are ongoing. We envisage future applications of the presented approach to inform and interpret diverse laser-field experiments on molecules containing nuclei with large quadrupole constants.

This work was supported by the Deutsche Forschungsgemeinschaft (DFG) through the priority program "Quantum Dynamics in Tailored Intense Fields" (QUTIF, SPP1840, KU 1527/3, YA $610 / 1)$ and the clusters of excellence "Center for Ultrafast Imaging" (CUI, EXC 1074, ID 194651731) and "Advanced Imaging of Matter" (AIM, EXC 2056, ID 390715994). L.V.T. would like to thank Rosario González-Férez for helpful discussions and for the hospitality at the University of Granada.

\section{REFERENCES}

${ }^{1}$ L. Christensen, J. H. Nielsen, C. B. Brandt, C. B. Madsen, L. B. Madsen, C. S. Slater, A. Lauer, M. Brouard, M. P. Johansson, B. Shepperson, and H. Stapelfeldt, "Dynamic Stark control of torsional motion by a pair of laser pulses," Phys. Rev. Lett. 113, 073005 (2014).

${ }^{2}$ B. Shepperson, A. A. Søndergaard, L. Christiansen, J. Kaczmarczyk, R. E. Zillich, M. Lemeshko, and H. Stapelfeldt, "Laser-induced rotation of iodine molecules in helium nanodroplets: Revivals and breaking free,” Phys. Rev. Lett. 118, 203203 (2017); e-print arXiv:1702.01977 [physics].

${ }^{3}$ E. T. Karamatskos, S. Raabe, T. Mullins, A. Trabattoni, P. Stammer, G. Goldsztejn, R. R. Johansen, K. Długołęcki, H. Stapelfeldt, M. J. J. Vrakking, S. Trippel, A. Rouzée, and J. Küpper, "Molecular movie of ultrafast coherent rotational dynamics of OCS," Nat. Commun. 10, 3364 (2019); e-print arXiv:1807.01034 [physics].

${ }^{4}$ C. P. Koch, M. Lemeshko, and D. Sugny, "Quantum control of molecular rotation,” Rev. Mod. Phys. 91, 035005 (2019).

${ }^{5}$ H. Stapelfeldt and T. Seideman, "Colloquium: Aligning molecules with strong laser pulses,” Rev. Mod. Phys. 75, 543-557 (2003).

${ }^{6}$ L. Holmegaard, J. H. Nielsen, I. Nevo, H. Stapelfeldt, F. Filsinger, J. Küpper, and G. Meijer, "Laser-induced alignment and orientation of quantum-state-selected large molecules," Phys. Rev. Lett. 102, 023001 (2009); e-print arXiv:0810.2307 [physics].

${ }^{7}$ O. Ghafur, A. Rouzée, A. Gijsbertsen, W. K. Siu, S. Stolte, and M. J. J. Vrakking, "Impulsive orientation and alignment of quantum-state-selected NO molecules," Nat. Phys. 5, 289-293 (2009).

${ }^{8}$ C. J. Hensley, J. Yang, and M. Centurion, "Imaging of isolated molecules with ultrafast electron pulses,” Phys. Rev. Lett. 109, 133202 (2012).
${ }^{9}$ J. Küpper, S. Stern, L. Holmegaard, F. Filsinger, A. Rouzée, A. Rudenko, P. Johnsson, A. V. Martin, M. Adolph, A. Aquila, S. Bajt, A. Barty, C. Bostedt, J. Bozek, C. Caleman, R. Coffee, N. Coppola, T. Delmas, S. Epp, B. Erk, L. Foucar, T. Gorkhover, L. Gumprecht, A. Hartmann, R. Hartmann, G. Hauser, P. Holl, A. Hömke, N. Kimmel, F. Krasniqi, K.-U. Kühnel, J. Maurer, M. Messerschmidt, R. Moshammer, C. Reich, B. Rudek, R. Santra, I. Schlichting, C. Schmidt, S. Schorb, J. Schulz, H. Soltau, J. C. H. Spence, D. Starodub, L. Strüder, J. Thøgersen, M. J. J. Vrakking, G. Weidenspointner, T. A. White, C. Wunderer, G. Meijer, J. Ullrich, H. Stapelfeldt, D. Rolles, and H. N. Chapman, "X-ray diffraction from isolated and strongly aligned gas-phase molecules with a free-electron laser," Phys. Rev. Lett. 112, 083002 (2014); e-print arXiv:1307.4577 [physics].

${ }^{10}$ J. Itatani, J. Levesque, D. Zeidler, H. Niikura, H. Pépin, J. C. Kieffer, P. B. Corkum, and D. M. Villeneuve, "Tomographic imaging of molecular orbitals," Nature 432, 867-871 (2004).

${ }^{11}$ T. Kanai, S. Minemoto, and H. Sakai, "Quantum interference during high-order harmonic generation from aligned molecules," Nature 435, 470-474 (2005).

${ }^{12}$ L. Holmegaard, J. L. Hansen, L. Kalhøj, S. L. Kragh, H. Stapelfeldt, F. Filsinger, J. Küpper, G. Meijer, D. Dimitrovski, M. Abu-samha, C. P. J. Martiny, and L. B. Madsen, "Photoelectron angular distributions from strong-field ionization of oriented molecules," Nat. Phys. 6, 428 (2010); e-print arXiv:1003.4634 [physics].

${ }^{13}$ F. Filsinger, G. Meijer, H. Stapelfeldt, H. Chapman, and J. Küpper, "Stateand conformer-selected beams of aligned and oriented molecules for ultrafast diffraction studies," Phys. Chem. Chem. Phys. 13, 2076-2087 (2011); e-print arXiv:1009.0871 [physics].

${ }^{14}$ S. J. Weber, M. Oppermann, and J. P. Marangos, "Role of rotational wave packets in strong field experiments," Phys. Rev. Lett. 111, 263601 (2013).

${ }^{15}$ E. W. Kuipers, M. G. Tenner, A. Kleyn, and S. Stolte, "Observation of steric effects in gas-surface scattering," Nature 334, 420-422 (1988).

${ }^{16}$ T. P. Rakitzis, A. J. van den Brom, and M. H. M. Janssen, "Directional dynamics in the photodissociation of oriented molecules," Science 303, 1852-1854 (2004).

${ }^{17}$ V. Aquilanti, M. Bartolomei, F. Pirani, D. Cappelletti, F. Vecchiocattivi, Y. Shimizu, and T. Kasai, "Orienting and aligning molecules for stereochemistry and photodynamics," Phys. Chem. Chem. Phys. 7, 291-300 (2005).

${ }^{18}$ P. M. Felker, J. S. Baskin, and A. H. Zewail, "Rephasing of collisionless molecular rotational coherence in large molecules," J. Phys. Chem. 90, 724-728 (1986).

${ }^{19}$ F. Rosca-Pruna and M. J. J. Vrakking, "Experimental observation of revival structures in picosecond laser-induced alignment of $\mathrm{I}_{2}$," Phys. Rev. Lett. 87, 153902 (2001).

${ }^{20}$ M. Spanner, S. Patchkovskii, E. Frumker, and P. Corkum, "Mechanisms of twocolor laser-induced field-free molecular orientation,” Phys. Rev. Lett. 109, 113001 (2012); e-print arXiv:1205.4383 [physics].

${ }^{21}$ K. Lin, I. Tutunnikov, J. Qiang, J. Ma, Q. Song, Q. Ji, W. Zhang, H. Li, F. Sun, X. Gong, H. Li, P. Lu, H. Zeng, Y. Prior, I. S. Averbukh, and J. Wu, "Alloptical field-free three-dimensional orientation of asymmetric-top molecules," Nat. Commun. 9, 5134 (2018); e-print arXiv:1803.07823 [physics].

${ }^{22}$ J. H. Nielsen, H. Stapelfeldt, J. Küpper, B. Friedrich, J. J. Omiste, and R. González-Férez, "Making the best of mixed-field orientation of polar molecules: A recipe for achieving adiabatic dynamics in an electrostatic field combined with laser pulses," Phys. Rev. Lett. 108, 193001 (2012); e-print arXiv:1204.2685 [physics].

${ }^{23}$ S. Trippel, T. Mullins, N. L. M. Müller, J. S. Kienitz, R. González-Férez, and J. Küpper, "Two-state wave packet for strong field-free molecular orientation," Phys. Rev. Lett. 114, 103003 (2015); e-print arXiv:1409.2836 [physics].

${ }^{24}$ T. Seideman, "Revival structure of aligned rotational wave packets," Phys. Rev. Lett. 83, 4971-4974 (1999).

${ }^{25}$ F. Rosca-Pruna and M. J. J. Vrakking, "Revival structures in picosecond laserinduced alignment of $\mathrm{I}_{2}$ molecules. II. Numerical modeling," J. Chem. Phys. 116, 6579 (2002).

${ }^{26}$ T. Seideman and E. Hamilton, "Nonadiabatic alignment by intense pulses. Concepts, theory, and directions," Adv. At., Mol., Opt. Phys. 52, 289-329 (2005).

${ }^{27}$ L. V. Thesing, J. Küpper, and R. González-Férez, “Time-dependent analysis of the mixed-field orientation of molecules without rotational symmetry," J. Chem. Phys. 146, 244304 (2017); e-print arXiv:1705.03225 [physics]. 
${ }^{28}$ L. V. Thesing, A. Yachmenev, R. González-Férez, and J. Küpper, "Laser-induced alignment of weakly bound molecular aggregates," Phys. Rev. A 98, 053412 (2018); e-print arXiv:1808.01206 [physics].

${ }^{29}$ E. F. Thomas, A. A. Søndergaard, B. Shepperson, N. E. Henriksen, and H. Stapelfeldt, "Hyperfine-structure-induced depolarization of impulsively aligned $I_{2}$ molecules," Phys. Rev. Lett. 120, 163202 (2018); e-print arXiv:1804.04416 [physics].

${ }^{30}$ B. Erk, R. Boll, S. Trippel, D. Anielski, L. Foucar, B. Rudek, S. W. Epp, R. Coffee, S. Carron, S. Schorb, K. R. Ferguson, M. Swiggers, J. D. Bozek, M. Simon, T. Marchenko, J. Küpper, I. Schlichting, J. Ullrich, C. Bostedt, D. Rolles, and A. Rudenko, "Imaging charge transfer in iodomethane upon $\mathrm{x}$-ray photoabsorption," Science 345, 288-291 (2014).

${ }^{31}$ K. Nass, L. Foucar, T. R. M. Barends, E. Hartmann, S. Botha, R. L. Shoeman, R. B. Doak, R. Alonso-Mori, A. Aquila, S. Bajt, A. Barty, R. Bean, K. R. Beyerlein, M. Bublitz, N. Drachmann, J. Gregersen, H. O. Jönsson, W. Kabsch, S. Kassemeyer, J. E. Koglin, M. Krumrey, D. Mattle, M. Messerschmidt, P. Nissen, L. Reinhard, O. Sitsel, D. Sokaras, G. J. Williams, S. Hau-Riege, N. Timneanu, C. Caleman, H. N. Chapman, S. Boutet, and I. Schlichting, "Indications of radiation damage in ferredoxin microcrystals using high-intensity X-FEL beams," J. Synchrotron Radiat. 22, 225-238 (2015).

${ }^{32}$ I. Nevo, L. Holmegaard, J. H. Nielsen, J. L. Hansen, H. Stapelfeldt, F. Filsinger, G. Meijer, and J. Küpper, "Laser-induced 3D alignment and orientation of quantum state-selected molecules," Phys. Chem. Chem. Phys. 11, 9912-9918 (2009); e-print arXiv:0906.2971 [physics].

${ }^{33}$ T. Takanashi, K. Nakamura, E. Kukk, K. Motomura, H. Fukuzawa, K. Nagaya, S. ichi Wada, Y. Kumagai, D. Iablonskyi, Y. Ito, Y. Sakakibara, D. You, T. Nishiyama, K. Asa, Y. Sato, T. Umemoto, K. Kariyazono, K. Ochiai, M. Kanno, K. Yamazaki, K. Kooser, C. Nicolas, C. Miron, T. Asavei, L. Neagu, M. Schöffler, G. Kastirke, X.-J. Liu, A. Rudenko, S. Owada, T. Katayama, T. Togashi, K. Tono, M. Yabashi, H. Kono, and K. Ueda, "Ultrafast Coulomb explosion of a diiodomethane molecule induced by an x-ray free-electron laser pulse," Phys. Chem. Chem. Phys. 19, 19707-19721 (2017).

${ }^{34}$ A. Yachmenev and J. Küpper, "Communication: General variational approach to nuclear-quadrupole coupling in rovibrational spectra of polyatomic molecules," J. Chem. Phys. 147, 141101 (2017); e-print arXiv:1709.08558 [physics].

${ }^{35} \mathrm{~A}$. Owens and A. Yachmenev, "RichMol: A general variational approach for rovibrational molecular dynamics in external electric fields," J. Chem. Phys. 148, 124102 (2018); e-print arXiv:1802.07603 [physics].

${ }^{36} \mathrm{~S}$. N. Yurchenko, W. Thiel, and P. Jensen, "Theoretical ROVibrational energies (TROVE): A robust numerical approach to the calculation of rovibrational energies for polyatomic molecules," J. Mol. Spectrosc. 245, 126-140 (2007).

${ }^{37}$ A. Yachmenev and S. N. Yurchenko, "Automatic differentiation method for numerical construction of the rotational-vibrational Hamiltonian as a power series in the curvilinear internal coordinates using the Eckart frame," J. Chem. Phys. 143, 014105 (2015).

${ }^{38}$ S. N. Yurchenko, A. Yachmenev, and R. I. Ovsyannikov, "Symmetry adapted ro-vibrational basis functions for variational nuclear motion calculations: TROVE approach," J. Chem. Theory Comput. 13, 4368 (2017); e-print arXiv:1708.07185 [physics].

${ }^{39}$ K. L. Chubb, A. Yachmenev, J. Tennyson, and S. N. Yurchenko, "Treating linear molecule HCCH in calculations of rotation-vibration spectra," J. Chem. Phys. 149, 014101 (2018)

${ }^{40} \mathrm{P}$. Coles, A. Owens, J. Küpper, and A. Yachmenev, "Hyperfine-resolved rotation-vibration line list of ammonia $\left(\mathrm{NH}_{3}\right)$," Astrophys. J. 870, 24 (2018); e-print arXiv:1809.06915 [physics].

${ }^{41}$ E. Hamilton, T. Seideman, T. Ejdrup, M. D. Poulsen, C. Z. Bisgaard, S. S. Viftrup, and H. Stapelfeldt, "Alignment of symmetric top molecules by short laser pulses," Phys. Rev. A 72, 043402 (2005).

${ }^{42}$ A. Rouzee, S. Guerin, V. Boudon, B. Lavorel, and O. Faucher, "Field-free onedimensional alignment of ethylene molecule," Phys. Rev. A 73, 033418-033419 (2006).

${ }^{43}$ A. Rouzee, S. Guerin, O. Faucher, and B. Lavorel, "Field-free molecular alignment of asymmetric top molecules using elliptically polarized laser pulses," Phys. Rev. A 77, 043412 (2008).
${ }^{44}$ S. Trippel, T. Mullins, N. L. M. Müller, J. S. Kienitz, J. J. Omiste, H. Stapelfeldt, R. González-Férez, and J. Küpper, "Strongly driven quantum pendulum of the carbonyl sulfide molecule," Phys. Rev. A 89, 051401(R) (2014); e-print arXiv:1401.6897 [quant-ph].

${ }^{45}$ M. Torrent-Sucarrat, J. M. Luis, and B. Kirtman, "Variational calculation of vibrational linear and nonlinear optical properties," J. Chem. Phys. 122, 204108 (2005).

${ }^{46}$ F. Weigend, F. Furche, and R. Ahlrichs, "Gaussian basis sets of quadruple zeta valence quality for atoms $\mathrm{H}-\mathrm{Kr}$," J. Chem. Phys. 119, 12753-12762 (2003).

${ }^{47} \mathrm{~F}$. Weigend and R. Ahlrichs, "Balanced basis sets of split valence, triple zeta valence and quadruple zeta valence quality for $\mathrm{H}$ to $\mathrm{Rn}$ : Design and assessment of accuracy," Phys. Chem. Chem. Phys. 7, 3297 (2005).

${ }^{48}$ K. A. Peterson, D. Figgen, E. Goll, H. Stoll, and M. Dolg, "Systematically convergent basis sets with relativistic pseudopotentials. II. Small-core pseudopotentials and correlation consistent basis sets for the post-d group 16-18 elements," J. Chem. Phys. 119, 11113-11123 (2003)

${ }^{49} \mathrm{D}$. Hait and M. Head-Gordon, "How accurate are static polarizability predictions from density functional theory? An assessment over 132 species at equilibrium geometry," Phys. Chem. Chem. Phys. 20, 19800-19810 (2018).

${ }^{50}$ F. Neese, A. Wolf, T. Fleig, M. Reiher, and B. A. Hess, "Calculation of electricfield gradients based on higher-order generalized Douglas-Kroll transformations," J. Chem. Phys. 122, 204107 (2005).

${ }^{51}$ F. E. Jorge, A. C. Neto, G. G. Camiletti, and S. F. Machado, "Contracted Gaussian basis sets for Douglas-Kroll-Hess calculations: Estimating scalar relativistic effects of some atomic and molecular properties," J. Chem. Phys. 130, 064108 (2009).

${ }^{52} \mathrm{C}$. Campos and F. Jorge, "Triple zeta quality basis sets for atoms Rb through Xe: Application in $\operatorname{CCSD}(\mathrm{T})$ atomic and molecular property calculations," Mol. Phys. 111, 167-173 (2012).

${ }^{53} \mathrm{R}$. Bjornsson and M. Bühl, "Electric field gradients of transition metal complexes: Basis set uncontraction and scalar relativistic effects," Chem. Phys. Lett. 559, 112-116 (2013).

${ }^{54}$ F. Neese, “The ORCA program system," Wiley Interdiscip. Rev.: Comput. Mol. Sci. 2, 73-78 (2011)

${ }^{55}$ F. Neese, "Software update: The ORCA program system, version 4.0," Wiley Interdiscip. Rev.: Comput. Mol. Sci. 8, e1327 (2017).

${ }^{56}$ P. Pyykkö, "Year-2008 nuclear quadrupole moments," Mol. Phys. 106, 19651974 (2008).

${ }^{57}$ F. Filsinger, J. Küpper, G. Meijer, L. Holmegaard, J. H. Nielsen, I. Nevo, J. L. Hansen, and H. Stapelfeldt, "Quantum-state selection, alignment, and orientation of large molecules using static electric and laser fields," J. Chem. Phys. 131, 064309 (2009); e-print arXiv:0903.5413 [physics].

${ }^{58}$ Y.-P. Chang, D. A. Horke, S. Trippel, and J. Küpper, "Spatially-controlled complex molecules and their applications," Int. Rev. Phys. Chem. 34, 557-590 (2015); e-print arXiv:1505.05632 [physics].

${ }^{59}$ S. Trippel, M. Johny, T. Kierspel, J. Onvlee, H. Bieker, H. Ye, T. Mullins, L. Gumprecht, K. Długołęcki, and J. Küpper, "Knife edge skimming for improved separation of molecular species by the deflector," Rev. Sci. Instrum. 89, 096110 (2018); e-print arXiv:1802.04053 [physics].

${ }^{60}$ M. D. Poulsen, E. Peronne, H. Stapelfeldt, C. Z. Bisgaard, S. Viftrup, E. Hamilton, and T. Seideman, "Nonadiabatic alignment of asymmetric top molecules: Rotational revivals,” J. Chem. Phys. 121, 783-791 (2004).

${ }^{61}$ L. Holmegaard, S. S. Viftrup, V. Kumarappan, C. Z. Bisgaard, H. Stapelfeldt, E. Hamilton, and T. Seideman, "Control of rotational wave-packet dynamics in asymmetric top molecules," Phys. Rev. A 75, 051403 (2007).

${ }^{62}$ A. Chatterley, E. T. Karamatskos, C. Schouder, L. Christiansen, A. V. Jörgensen, T. Mullins, J. Küpper, and H. Stapelfeldt, "Switched wave packets with spectrally truncated chirped pulses," J. Chem. Phys. 148, 221105 (2018); e-print arXiv:1803.03953 [physics].

${ }^{63}$ W. Gordy and R. L. Cook, Microwave Molecular Spectra, 3rd ed. (John Wiley \& Sons, New York, NY, USA, 1984). 